\title{
EDINBURGH MATHEMATICAL SOCIETY
}

\section{LIST OF MEMBERS-January 1936.}

\section{Honorary Members.}

H. F. BAKER, M.A., LL.D., F.R.S., Lowndean Professor of Astronomy and Geometry in the University of Cambridge, 3 Storey's Way, Cambridge

G. D. Birkhoff, Ph.D., Professor of Mathematics, Harvard University, Cambridge, Mass., U.S.A.

T. Levi-Civita, Professor of Mathematics in the University of Rome, Via Sardegna 5o, Rome (25), Italy

R. Franklin Muirhead, B.A., D.Sc., 8 Park Avenue, Glasgow, C. 3

H. W. Rrchmond, M.A., LL.D , F.R.S., King's College, Cambridge

D'Arcy W. Thompson, C.B., D.Sc., D.Litt., F.R.S., Professor of Natural History in the University of St Andrews, 44 South Street, St Andrews

\section{Ordinary Members.}

E. P. Adams, Ph.D., Professor of Physics, Princeton University, New Jersey, U.S.A. John R. Airey, M.A., D.Sc., Llwynon, Newtown, Montgomeryshire

A. C. ArtKen, M.A., D.Sc., F.R.S.E., Mathematical Institute, The University, Edinburgh

V. Ramaswami Aiyar, M.A., Retired Deputy Collector, Chittoor, S. India

5 John Alison, M.A., LL.D., F.R.S.E., 126 Craiglea Drive, Edinburgh, io

Albert Anderson, M.A., B.Sc., 272 Croftpark Avenue, Glasgow, S.4

James Anderson, M.A., Ph.D., 48 Greendykes Road, Dundee

William ArThur, M.A., F.R.S.E., Lecturer in Mathematics in Glasgow University, 148 Carmunnock Road, Cathcart, Glasgow

Bevan B. Baker, M.A., D.Sc., F.R.S.E., Professor of Mathematics in the University of London, Pentlands, Englefield Green, Surrey

Io John Ballantine, M.A., Redding School, Falkirk

W. BARRETT, M.A., H.M. Inspector of Factories, Fuglestemmen, Layters Way, Gerrards Cross, Bucks

F. Bath, B.Sc., Ph.D., F.R.S.E., University College, Dundee (Co-Editor of Procedings)

Sir J. C. Beattie, D.Sc., LL.D. F.R.S.E., Vice-Chancellor and Principal of the University of Cape Town, S. Africa

Archibald Bell, B.Sc., Longmuirhead, Lenzie

15 Robt. J. T. Bell, M.A., D.Sc., LL.D., F.R.S.E., Professor of Mathematics, University of Otago, Dunedin, New Zealand.

Peter Bennett, i4 Cecil Street, Hillhead, Glasgow

R. B. Bennetr, M.A., B.Sc., 2 Chesser Avenue, Edinburgh, II

W. E. H. Berwick, M.A., Professor of Mathematics, University College of North Wales; Ceinwen, Upper Bangor, North Wales

C. K. Bird, M.A., Assistant Goods Manager, L.N.E.R., King's Cross Station, London, N. I

20 F. P. Bisacre, M.A, c/o Messrs Blackie \& Co., 17 Stanhope Street, Glasgow

S. B. Bishara, B.Sc., Royal Engineering School, Guiza, Egypt

T. P. Black, M.A., B.Sc., Ph.D., F.R.S.E., Headmaster, Bellevue School, Edinburgh 
A. W. Boldyreff, B.S., M.S., Ph.D., Assistant Professor of Mathematics, University of Arizona, Tusson, Arizona, U.S.A.

Edward Blades, M.A., B.Sc., I Lockharton Crescent, Edinburgh, 1 I

25 F. Bradshaw, M.A, D.Sc., The Library, Armstrong College, Newcastle-upon-Tyne

RaYmond W. Brink, Ph.D., Professor of Mathematics, University of Minnesota, Minneapolis, Minnesota, U.S.A.

S. Brodetsky, Ph.D., Professor of Applied Mathematics, The University, Leeds

A. Brown, M.A., B.Sc., F.R.S.E., Professor of Applied Mathematics, University of Cape Town, S. Africa

F. G. W. Brown, M.Sc., F.C.P., 25 Welwood Road, Goodmayes, Essex

30 George Brown, M.A., 23 Gates Road, Lochwinnoch, Renfrewshire

John T. Brown, M.A. B.Sc., High School, North Berwick

L. M. Brown, M.Sc., Royal Technical College, Glasgow, C. I (Editor of Mathematical Notes)

T. A. Brown, M.A., B.Sc. F.R.S.E., Professor of Mathematics, University College, Exeter

Walter Brown, M.A., B.Sc., F.R.S.E., Professor of Mathematics, University of Hong-Kong, China

35 Thomas Brydone, M.A., B.Sc., The High School, West Calder

A. F. Buchan, B.Sc., James Gillespie's High School for Girls, Edinburgh

J. Buchanan, M.A., Perse School, Cambridge

J. W. Butters, M.A., B.Sc., F.R.S.E., 116 Comiston Drive, Edinburgh, to

Miss Nora Calderwood, M.A., B.Sc., The University, Edgbaston, Birmingham

40 John Campbell, B.Sc., North Kelvinside Secondary School, Oban Drive, Glasgow, N.W.

J. T. Campbell, M.A., Ph.D., Nelson College, Nelson, New Zealand.

George A. Carse, M.A., D.Sc., F.R.S.E., Reader in Natural Philosophy, The University, Edinburgh, I

H. S. Carslaw, Sc.D., LL.D., F.R.S.E., Emeritus Professor of Mathematics, The University, Sydney, N.S.W.

J. Cassels, M.A., B.Sc., The Academy, Ayr

45 G. E. Chappell, M.A., County School, Bridgend, Glamorgan

C. B. ChILds, Ph.D., Lecturer in Natural Philosophy, The University, Edinburgh, I

N. L. Clapton, M.A., 47 Cranworth Street, Hillhead, Glasgow, C.2

H. Clements, B.A., The Nautical College, Leith

Leo J. Close, M.A., Inspector of Schools, I Hume Street, Dublin

50 Rev. J. Riversdale Colthurst, M.A., Calary Rectory, Kilpedder, Co. Wicklow, Ireland

Peter Comrie, M.A., B.Sc., LL.D., F.R.S.E., 19 Craighouse Terrace, Edinburgh, to

Miss J. B. Constable, M.A., B.Sc., 3o Wilton Hill, Hawick

E. T. Copson, M.A., D.Sc., F.R.S.E., Professor of Mathematics, University College, Dundee (Co-Editor of Proceedings)

J. Cossar, M.A., Queen Mary College, Mile End Road, London, E. I

55 James I. Craig, M.A., F.R.S.E., Financial Secretary to the Egyptian Government ; Turf Club, Cairo, Egypt

G. E. Crawford, M.A., Barrister-at-Law, 6 Fig Tree Court, Temple, London, E.C. 4

Lawrence Crawford, M.A., D.Sc., F.R.S.E., Professor of Pure Mathematics, University of Cape Town, S. Africa

L. B. C. Cunningham, M.A., Ph.D., 38 Buckingham Terrace, Edinburgh, 4

J. P. Dalton, M.A., D.Sc., Professor of Mathematics, The University of the Witwatersrand, P.O. Box 1176 , Johannesburg, South Africa

60 Charles G. Darwin, M.A., F.R.S., Tait Professor of Natural Philosophy, The University, Edinburgh, I

E. T. Davies, Ph.D., M.Sc., King's College, London, W.C. 2

Miss Winifred Deans, M.A., B.Sc., c/o Messrs Blackie \& Son, Ltd., 17 Stanhope Street, Glasgow, C. 4 . 
Jacques Devisme, D. ès Sc., Professeur Agrégé de Mathématiques Spéciales, Lycée de Garçons, Tours, Indre et Loire, France

S. C. Dhar, D.Sc., Head of the Department of Mathematics, College of Science, Nagpur, India

65 A. C. Dixon, M.A., F.R.S., Emeritus Professor of Mathematics, Queen's University, Belfast; Malone, St. Mary's Avenue, Northwood, Middlesex

James M. Doggart, B.A., 47 Upper Clapton Road, London, E. 5

John Dougall, M.A., D.Sc., F.R.S.E., 47 Airthrey Avenue, Glasgow, W. 4

J. B. Dow, M.A., Standard Life Insurance Co., 3 George Street, Edinburgh, 2

David Drysdale, M.A., High School, Arbroath

70 Robert T. Dunbar, M.A., B.Sc., Ph.D., Professor of Physics, University College, Cardiff

George S. Eastwood, B.Sc., F.R.S.E., Craigie Lea, Cowdenbeath, Fife

W. L. EdGE, M.A., F.R.S.E., Mathematical Institute, 16 Chambers Street, Edinburgh, I John S. Edwards, M.A., Fettes College, Edinburgh

M. D. W. Elphinstone, Southchurch Rectory, Southend-on-Sea, Essex

75 I. M. H. Etherington, B.A., Ph.D., F.R.S.E., Mathematical Institute, 16 Chambers Street, Edinburgh, I (Hon. Secretary)

W. L. Ferrar, M.A., F.R.S.E., Fellow and Tutor of Hertford College, Oxford

James H. Filshie, M.A., B.Sc., Hillhead High School, Glasgow

Lester R. Ford, M.A., Pl..D., Professor of Mathematics, Rice Institute, Houston, Texas, U.S.A.

Ronald M. Foster, American Telephone and Telegraph Co., 195 Broadway, New York, U.S.A.

8o D. Franklin, M.Sc., Mathematical Institute, 16 Chambers Street, Edinburgh, I

David Kennedy-Fraser, M.A., B.Sc., Edge o' the Moor, Milngavie, Dumbartonshire

Peter Fraser, M.A., Reader in Geometry, The University, Bristol

G. Frecheville, M.A., B.Sc., Windmill Hill Farm, Steeple Claydon, Bucks.

G. L. Frewin, M.A., Reay House, Old Edinburgh Road, Inverness

85 J. D. Fulton, M.A., B.Sc., Edinburgh Ladies' College, Queen Street, Edinburgh, 2

J. P. Gabbatt, M.A., Durford Edge, Petersfield, Hants

Samuel Gedues, M.A., B.Sc., 183 Loanfoot Avenue, Glasgow, W. 3

DAvid GibB, M.A., B.Sc., F.R.S.E., Reader in Mathematics, Mathematical Institute, The University, Edinburgh, I. (Hon. Librarian)

C. Gilbert, B.Sc., Ph.D., Department of Mathematics, University College, Dundee

90 R. P. Gillespie, M.A., B.Sc., Ph.D., F.R.S.E., MacBrayne Hall, Park Circus Place, Glasgow, C. 3

H. T. Gonin, M.Sc., Ph.D., Stellenbosch, Cape Colony, S. Africa

P. G. Gormley, M.A., Ph.D., Carnanban, Claudy, Co. Derry, N. Ireland

Thos. S. Graham, M.A., Ph.D., 112 Nithsdale Road, Glasgow, S. I

Miss Marion C. Gray, M.A., Ph.D., c'o American Telephone \& Telegraph Co., D. \& R. Dept., 195 Broadway, New York City, U.S.A.

95 A. B: Grieve, M.A., D.Sc., H.M.I.S., Elmbank, Strathpeffer, Ross-shire

Hansraj Gupta, M.A., Professor of Mathematics, Government College, Hoshiarpur, Punjab, India

P. N. Das Gupta, M.Sc., Ph.D., Professor of Mathematics, Science College, Patna, India

W. G. Guthrie, M.A., Ph.D., F.R.S.E., Professor of Mathematics, McCrea-Magee College, Londonderry, N. I reland

R. L. Gwilt, F.I.A., F.F.A., c/o Scottish Widows' Fund, 9 St Andrew Square, Edinburgh, 2

100 C. D. Hardie, M.A., 98 Ormonde Crescent, Muirend, Glasgow, S. 4

J. A. Hardie, M.A., B.Sc., Daniel Stewart's College, Edinburgh, 4

P. S. Hardie, M.A., B.Sc., F.R.S.E., 19 Ardmillan Terrace, Edinburgh, 1 I 
R. P. Hardie, M.A., I3 Palmerston Road, Edinburgl, 9

Miss F. M. Harding, M.A., Ph.D., Astell, Overton Park Road, Cheltenham, Glos.

105 G. H. B. Haskard, B.Sc., South Australian School of Mines and Industries, North Terrace, Adelaide, S. Australia

T. Hayashi, Professor of Mathematics, Tohoku University, Sendai, Japan

H. A. HaYden, D.Sc., M.Sc., Head of Mathematics Department, Battersea Polytechnic, London, S.W. I I

J. W. Head, B.A., Canford School, Wimborne, Dorset

Alex. Henderson, M.A., High School, Dunfermline

I 10 J. Henderson, M.A., Ph.D., Lecturer in Mathematics, King's College, London, W.C. 2

Hugh Higgins, M.A., B.Sc., 6i First Avenue, Netherlee, Glasgow, S. 4

G. Hislop, M.A., B.Sc., 20 South Dewar Street, Dunfermline

W. A. Honohan, M.A., 3 Annesley Bridge Road, Fairview, Dublin

A. E. Hope, M.A., B.Ed., The Academy,'Forres, Morayshire

II 5 Pierre Humbert, Professeur à la Faculté des Sciences, Université de Montpellier, France

H. Hussein, M.A., Department of Mathematics, The University, Leeds

James Hyslop, M.A., Ph.D., F.R.S.E., Lecturer in Mathematics, The University, Glasgow, W. 2

J. M. Hyslop, 28 Bellwood Street, Langside, Glasgow, S. I

E. L. INCE, M.A., D.Sc., F.R.S.E., Mathematical Institute, I6 Chambers Street, Edinburgh, I

I 20 Alex. Inglis, M.Sc., Ph.D., Bell Baxter School, Cupar, Fife

W. M. Inverarity, M.A., B.Sc., F.R.A.S., 46 Stamperland Gardens, Clarkston, Renfrewshire

David JACK, M.A., B.Sc., Ph.D., F.R.S.E., Lecturer in Natural Philosophy, The University, St Andrew's

Grorge Johnston, M.A., Speir's School, Beith

H. A. Jordan, Ph.D., Lecturer in Mathematics, Georgetown University, Washington, D.C., U.S.A.

125 G. R. Keating, M.Sc., Glencar, Marlboro' Road, Glenageary, Co. Dublin

W. O. Kermack, M.A., D.Sc., F.R.S.E., Laboratory of the Royal College of Physicians, 2 Forrest Road, Edinburgh, I

J. E. Kerrich, M.Sc., The Department of Mathematics, The University of the Witwatersrand, Johannesburg, South Africa

W. F. Kibble, M.A., II Strathearn Place, Edinburgh, to

C. G. Lambe, B.A., Ph.D., Lecturer in Mathematics, Royal Military Academy, Woolwich, London, S.E. 18

130 Archibald Lang, M.A., B.Sc., Speybank, 52 Crown Drive, Inverness

George Lawson, M.A., 49 Merchiston Crescent, Edinburgh, ro

W. Ledermann, Department of Mathematics, The University, St Andrews

Charles A. Lee, M.A., Robert Gordon's College, Aberdeen

H. C. LeE, B.Sc., Mathematical Institute, I6 Chambers Street, Edinburgh, I

135 Alexander Leighton, M.A., B.Sc., LL.D., O.B.E., 4I Strathern Road, West Ferry, Dundee

H. Levy, M.A., D.Sc., F.K.S.E., Professor of Mathematics, Imperial College of Science and Technology, South Kensington, London, S.W.7

G. J. Lidstone, LL.D., F.I.A., F.F.A., F.R.S.E., Hermiston House, Hermiston, Currie, Midlothian

N. M. H. LightFoot, M.A., F.R.S.E., The Heriot Watt College, Edinburgh, I

J. B. Lockhart, M.A., B.Sc., F.R.S.E., Dundas House, Kinnear Road, Edinburgh, 4 (Hon. Treasurer)

140 WM. H. Love, M.A., Provincial Training Centre, Jordanhill, Glasgow

Thomas A. Lumsden, M.A., B.Sc., Lecturer in Mathematics, The University, Edgbaston, Birmingham

NeIL McArthUR, M.A., B.Sc., F.R.S.E., Lecturer in Mathematics in the University of Glasgow, I Holyrood Crescent, Glasgow 
Miss Eve Macbean, M.A., 2o Warrender Park Crescent, Edinburgh, ro James A. McBride, B.A., B.Sc., F.R.S.E., Queen's Park School, Glasgow

145 J. P. McCarthy, M.A., Department of Mathematics, University of Queensland, Brisbane, Australia

W. H. M'Crea, M.A., Ph.D., F.R.A.S., Assistant Professor of Mathematics, Imperial College of Science and Technology, South Kensington, London, S.W.7

Miss G. E. MacDonald, M.A., 3 Wendover Crescent, Mount Florida, Glasgow

James A. Macdonald. M.A., B.Sc., F.R.S.E., H.M.I.S., Rothes, Frankscroft, Peebles

Rex Macdonald, M.A., The Academy, Dollar

iso D. C. McIntosh, M.A., D.Sc., F.R.S.E., Glenavon, Boat-of-Garten, Invernesshire

Miss Jeannie G. M'Intosh, M.A., Pine Cottage, Meigle, Perthshire

Col. A. G. M'Kendrick, M.D., F.R.C.P.E., F.R.S.E., Laboratory of the Royal College of Physicians, Forrest Road, Edinburgh, 1

John Mackie, M.A., D.Sc., F.R.S.E., I9 Beresford Avenue, Leith, Edinburgh, 5

John M'Kinnell, M.A., B.Sc., Professor of Mathematics, Natal University College, Pietermaritzburg, South Africa

155 Angus M'Lean, B.Sc., C.E., Principal, Technical College, Paisley

A. J. Maclean, M.A., B.A., 39 Mains Loan, Dundee

John Maclean, M.A., B.Sc, Professor of Mathematics, Wilson College, Bombay

D. B. M'Quistan, M.A., B.Sc., F.R.S.E., 29 Viewpark Drive, Rutherglen

Thomas M. MacRobert, M.A., D.Sc., F.R.S.E., Professor of Mathematics, to The University, Glasgow, W. 2

ı60 G. C. McVirtie, M.A., Ph.D., Department of Applied Mathematics, The University, Liverpool

John M'Whan, M.A., Ph.D., 84 Munro Road, Jordanhill, Glasgow, W. 3

J. B. Marshall, M.A., ro Corstorphine Road, Murrayfield, Edinburgh, 12

WM. Marshall, M.S., Ph.D., Head of Department of Mathematics, Purdue University, Lafayette, Indiana, U.S.A.

David B. Meek, M.A., D.Sc., Director of Industries (Bengal), Calcutta, India

165 J. B. Meiklejohn, M.A., Crescent Bank, East Newport, Fife

J. M. Meiklejohn, M.A., 12 Wyvis Terrace, Dingwall

A. S. Merrill, Ph.D, The Mathematics Department, The University of Montana, Missoula, Mont., U.S.A.

W. H. Metzler, A.B., D.Sc., F.R.S.E., formerly Professor of Mathematics at New York State College for Teachers, 4 Glenwood Street, Albany, N.Y., U.S.A.

Rev. George S. Mill, M.A., B.Sc., Universities' Mission, Kalimpong, Bengal, India

170 A. H. Miller, M.A., B.Sc., Preston Park, Prestonpans, East Lothian

John Miller, M.A., D.Sc., F.R.S.E., formerly Professor of Mathematics, Royal Technical College, Glasgow, 2 I 2 Wilton Street, Glasgow, N.W.

T. Hugh Miller, M.A., D.Sc., Lansdowne, Thurlow Road, Nairn

William Miller, M.A., Ph.D., D.Sc., Postlagernd, Klosterneuberg, Postamt 3 , Austria

Archibald Milne, M.A., D.Sc., F.R.S.E., Depute Director, Provincial Training College, Edinburgh

175 Edward L. Milne, M.A., B.Sc, 22 Church Street, Alloa

John Milne, M.A., Training Centre, Aberdeen

W. P. Milne, M.A., D.Sc., Professor of Mathematics, The University, Leeds

James Mitchell, M.A., B.Sc., F.R.S.E., Islay Lodge, Lochgilphead, Argyll

S. C. Mirra, Ph.D., Lecturer in Mathematics, University of Dacca, Dacca, Bengal. India

180 Radha Mohan, B.A., Sahu Mohalla, Moradabad (U.P.), India

Miss A. Mildred Moore, M.A., c/o Benson, 45A George Street, Edinburgh, 2 
W. More, M.A., Preston Lodge School, Prestonpans

Miss Isabella M. Morrison, M.A., B.Sc., 5 Kilmaurs Terrace, Edinburgh, 9

J. T. Morrison, M.A., B.Sc., F.R.S.E., Professor of Physics, Stellenbosch, Cape Colony, S. Africa

185 F. D. Murnaghan, M.A., Ph.D., Professor of Applied Mathematics, The Johns Hopkins University, Baltimore, Md., U.S.A.

M. Mursi-Ahmed, B.Sc., Ph.D., Faculty of Science, Egyptian University, AbbasiahCairo, Egypt

Jason J. Nassau, C.E., M.Sc., Director of the Warner and Swasey Observatory, Case School of Applied Science, Cleveland, Ohio, U.S.A.

C. A. Noble, Ph.D., Professor of Mathematics in the University of California, 2224 Piedmont Avenue, Berkeley, California, U.S.A.

G. Oliver, M.A., High School, Dunfermline

rgo A. Oppenherm, B.A., Ph.D., Professor of Mathematics,.' Raffles College, Singapore James Paton, M.A., B.Sc., The Natural Philosophy Department, The University, Edinburgh, I

James Paul, M.A., B.Sc., Royal Technical College, Glasgow

G. S. Pearson, M.A., The Academy, Alloa

Wm. Peddie, D.Sc., F.R.S.E., Professor of Natural Philosophy, University College, Dundee

195 Miss E. B. Philip, M.A., The Mary Acre, Brechin

George Philip, M.A., D.Sc., Education Offices, Dingwall

Wm. Edward Philip, M.A., H.M.I.S., 3 North Charlotte Street, Edinburgh, 2

Edgar G. Phillips, M.A., M.Sc., Danesbury, Craig-y-don Road, Bangor, N. Wales

Henry T. H. Piaggio, M.A., D.Sc., Professor of Mathematics, University College, Nottingham

200 D. K. Picken, M.A., Master of Ormond College, University of Melbourne, Victoria

R. H. Pinkerton, M.A., Emeritus Professor of Mathematics, University College, Cardiff

Balth. van Der Pol, D.Sc., Parklaan 24, Eindhoven, Holland

GorakH Prasad, D.Sc., Reader in Mathematics, University of Allahabad, Allahabad, India

Edward Press, Bank of Ethiopia, Addis Ababa, Ethiopia

205 A. T. Price, M.Sc., Crossways, Lees Road, Wellingdon, Middlesex

D. G. Ramsay, M.A., B.Sc., The Academy, Kirkcudbright

Thos. T. Rankin, C.E., B.Sc., I Nether Park, Finchley, London, N. 3

S. READ, B.A., F.R.S.E., 2 Oxford Terrace, Edinburgh, 4

G. W. Rispon, i I Victoria Street, London, S.W. I

210 J. C. Ritchie, M.A., Darroch Intermediate School, Edinburgh

R. A. Roвb, M.A., M.Sc., F.R.S.E., Department of Mathematics, The University, Glasgow

James Robertson, M.A., B.Sc., The Provincial Training College, Glasgow

George Robinson, M.A, B.Sc., $54^{2}$ Fourteenth Avenue W., Calgary, Alberta, Canada

H. F. Rose, M.A., Brackenbrae, I Northfield, Edinburgh, 9

215 Edward B. Ross, M.A., F.R.S.E., 4I Liberton Brae, Edinburgh, 9

C. H. Rowe, M.A., F.T.C.D., Professor of Mathematics, Trinity College, Dublin

A. S. Roy, B.Sc., 22 Shamrock Street, Dundee

H. S. Ruse, M.A., D.Sc., F.R.S.E., Mathematical Institute, The University, Edinburgh, I (President and Co-Editor of Proceedings)

Alex. D. Russell, B.Sc., F.R.S.E., F.R.A.S., I4 Heugh Street, Falkirk

220 D. E. Rutherford, M.A., B.Sc.. Dr in Wis- en Natuurkunde, F.R.S.E., Department of Mathematics, The University, St Andrews

William Saddler, M.A., D.Sc., F.R.S.E., Professor of Mathematics, Canterbury College, Christchurch, New Zealand

K. J. Sanjana, Professor of Mathematics, Bhavnagar, Bombay 
Robert Schlapp, M.A., Ph.D., F.R.S.E., The Natural [Philosophy Department, The University, Edinburgh, I

R. J. Schmidt, B.Sc., Ph.D., Imperial College of Science and Technology, S. Kensington, London, S.W. 7

225 Miss S. Scort, M.A., St Leonard's School, St Andrews, Fife

Thos. Scotr, M.A., Inch of Ferryton, Clackmannan

T. J. J. SEE, M.A., Ph.D., Professor of the U.S. Naval Observatory, Mare Island, California, U.S.A.

C. J. Seelye, M.Sc., Mathematical Institute, The University, Edinburgh, I

J. G. Semple, B.A., Ph.D., Professor of Mathematics, Queen's University, Belfast

230 R. N. Sen, M.A., Ph.D., Department of Mathematics, The University, Calcutta, India

N. G. Shabde, M.A., FD.Sc., Professor of Mathematics, City College, Nagpur City, India

Roderick M. Shearer, M.A., B.Sc., 14 Ross Gardens, Edinburgh, 9

W. F. Sheppard, Sc.D., Ll.M., F.R.S.E., Cardrona, Berkhampsted, Herts

Miss Catherine W. M. Sherriff, M.A., B.Sc., High Beveridgewell, Dunfermline

235 E. F. Simonds, B.Sc., Ph.D., Professor of Mathematics, University of Queensland, Brisbane, Australia

N. B. Slater, M.A., Caius College, Cambridge

Glenny Smeal, B.Sc., Lecturer in Mathematics, The University, Leeds

J. H. Smith, B.Sc., Leith Nautical College, Leith

J. H. Sмгтн, M.A., I79 Knightswood Road, Glasgow, W. 3

240 R. A. Smith, M.A., Department of Mathematics, United College, St Andrews

C. A. Stewart, M.A., B.Sc., The University, Sheffield

James W. Stewart, B.A., Lyme Cottage, Kelso

W. J. van Stockum, M.A., Mathematical Institute, 16 Chambers Street, Edinburgh

George D. C. Stokes, M.A., D.Sc., St Ruth's, Maxwelltown, Dumfries

245 Chas. Strachan, M.A., Ph.D., 213 Forest Avenue, Aberdeen

J. A. Strang, M.A., Professor of Mathematics, Canning College, Lucknow, India

R. O. Street, M.A., D.Sc., Professor of Mathematics, Royal Technical College, Glasgow, C. I (Vice-President)

David G. TAYlor, M.A., D.Sc., Lecturer in Mathematics, University College, Cardiff

William Taylor, M.A., Moray House Training College, Edinburgh

250 J. A. Third, M.A., D.Sc., 6 St Vincent Street, Edinburgh, 3

George Thomson, M.A., Avondale, Clyde Street, Carluke

James Thomson, M.A., D.Sc., Robert Gordon's College, Aberdeen

Sir WM. Thomson, M.A., B.Sc., LL.D., F.R.S.E., Dunedin, Glencairn, Simonstown, South Africa

W. L. Thomson, M.A., Casita, 65 Traquair Park West, Edinburgh, 22

255 G. Timms, Ph.D., F.R.S.E., Department of Mathematics, The University, St Andrews

J. R. Timoney, M.A., Belleek, Co. Fermanagh, N. Ireland

J. A. Todv, Pl..D., Hulme Hall, Victoria Park, Manchester, 14

H. W. Turnbull, M.A., F.R.S., Regius Professor of Mathematics in the University of St Andrews, Randa, Hepburn Gardens, St Andrews, Fife

John Turner, M.A., B.Sc., I Io Findhorn Place, Edinburgh, 9

260 Sheriff George D. Valentine, M.A., Bronville, Kinnoull, Perth

R. S. Varma, M.Sc., Christ Church College, Cawnpore, India

Mrs Agnes M. Waddell, M.A., B.Sc.,

A. G. Walker, M.A., Ph.D., 55 Whippendell Road, Watford, Herts

Robert Walker, M.A., Provincial Training College Glasgow

265 C. E. Walsh, 34 Chelmsford Road, Ranelagh, Dublin

G. N. Watson, Sc.D., F.R.S., Professor of Mathematics, The University, Birmingham 
JAmes WATt, LL.D., W.S., F.F.A., F.R.S.E., 7 Blackford Road, Edinburgh, to

C. E. Weatherburn, M.A., D.Sc., Professor of Mathematics, The University, Perth, West Australia

K. K. Weatherhead, M.A., F.F.A., 28 St Andrew Square, Edinburgh, 2

270 J. H. Maclagan Wedderburn, M.A., D.Sc., F.R.S., Professor of Mathematics in Princeton University; Fine Hall, Princeton, N.J., U.S.A.

G. J. Whitrow, B.A., 37 Cedars Road, Clapham, London, S.W. 4

E. T. WhitTaker, Sc.D., LL.D., F.R.S., Professor of Mathematics, Mathematical Institute, The University, Edinburgh, I

J. M. Whittaker, M.A., D.Sc., F.R.S.E., Professor of Pure Mathematics, The University, Liverpool

J. Williamson, M.A., Ph.D., F.R.S.E., Professor University, Baltimore, Md., U.S.A.

275 R. WiLson, M.A., University College, Swansea, Glam.

J. R. Wilton, Sc.D. (Cantab), D.Sc. (Adelaide), Professor of Mathematics in the University of Adelaide, South Australia

C. E. Winn, M.A., Egyptian University, Abbasiah, Cairo, Egypt

Aurel Wintner, Ph.D., Associate Professor of Mathematics, The Johns Hopkins University, Baltimore, Maryland, U.S.A.

E. Maitland Wright, M.A., D.Phil,, Professor of Mathematics, The University, Aberdeen

280 Thomas Wylie, M.A., C.A., 17 Duke Street, Edinburgh, I

A. W. Young, M.A., B.Sc., LL.B., W.S., 4 Admiral Terrace, Edinburgh, to

Miss Edith Young, M.A., B.Sc., Stronvar, Larbert, Stirlingshire

Miss J. M. Younger, M.A., Hutcheson's Girls' Grammar School, Glasgow

G. Udny Yule, C.B.E., F.R.S., M.A., St John's College, Cambridge

285 M. ZIA UD DiN, M.A., University College, Swansea, S. Wales 